\title{
Psoriasis, Vitiligo, and Biologic Therapy: Case Report and Narrative Review
}

\author{
Martina Burlando Andrea Muracchioli Emanuele Cozzani \\ Aurora Parodi \\ Section of Dermatology, DISSAL, University of Genoa, Ospedale-Policlinico San Martino, \\ IRCCS, Genova, Italy
}

\section{Keywords}

Psoriasis · Vitiligo · Biologic therapy $\cdot$ Narrative review

\begin{abstract}
Psoriasis and vitiligo are 2 multifactorial immune-mediated diseases, partially sharing pathogenetic underpinnings. Their coexistence in the same patient, although uncommon, is documented in the literature. Further, several cases of vitiligo induced by biological drugs in psoriatic patients are reported. However, improvements in psoriasis and pre-existing vitiligo after the introduction of biological therapy are also described. To date, anti-TNF-alpha is the most cited group of biological drugs that induce new-onset vitiligo or progression of pre-existence vitiligo in psoriatic patients. Even anti-IL-12/23 class would seem to induce vitiligo (as in our case) or even worse it. Anti-IL-17 drugs induce a progression of pre-existing vitiligo while, to date, no cases are reported in literature considering anti-IL-23 class.
\end{abstract}

\section{Introduction}

Several cases of vitiligo induced by biological drugs in psoriatic patients are reported in the literature. However, improvements of pre-existing vitiligo after the introduction of biological therapy are also described. This relationship is not still clear. Based on the case of a young Italian male suffering from psoriasis, who developed a progression of a pre-existence vitiligo after treatment with ustekinumab, we explore the association between psoriasis, vitiligo, and biological therapy. We evaluated all the cases and studies reported in the literature, elaborating a narrative review to define, to date, which biological drugs are more involved in the progression/onset of vitiligo in the psoriatic patient. 
Fig. 1. Patches of vitiligo under the left axillary area appeared in November 2012. These lesions have remained unchanged until today.

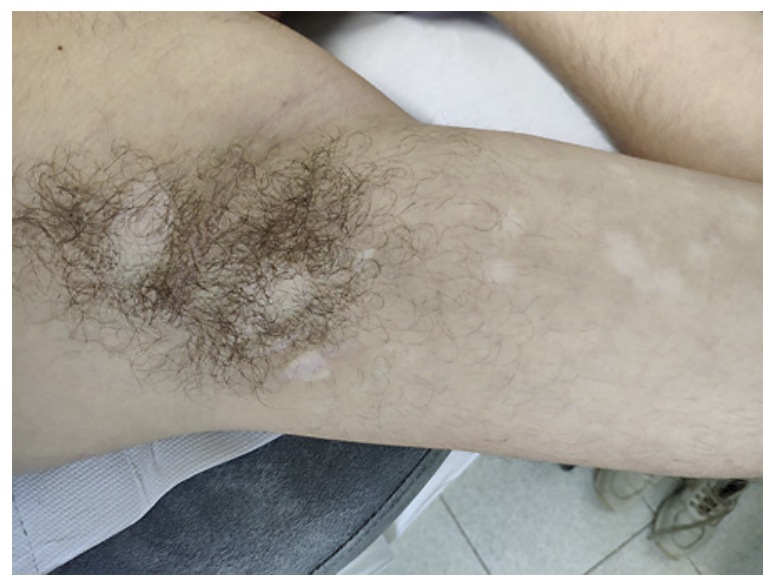

\section{Case Report}

A 32-year-old male outpatient came for a visit in April 2008 in our Dermatology department for the worsening of his psoriasis. He has suffered from plaque psoriasis, localized at the genitalia, elbows, and scalp, since the age of 11 . The patient was treated with various topical therapies without success and followed up regularly. In November 2012 , vitiligo patches appeared in the subaxillary area and subsequently on the perioral area (shown in Fig. 1). The thyroid function was investigated and was normal; furthermore, celiac disease was excluded. Therapy with methotrexate $(10 \mathrm{mg} /$ week IM) followed by folic acid $24 \mathrm{~h}$ later was started in February 2016. After 24 months, it was suspended for intolerance and inefficacy. In April 2018, his clinical state worsened. The Psoriasis Area and Severity Index (PASI) was 15, while the hypopigmented patches were stable. Having appropriate screening exams, he started biologic therapy with ustekinumab (Stelara ${ }^{\circledR}$ ) $90 \mathrm{mg}$ SC (followed by injection after 4 weeks and every 12 weeks for maintenance). His psoriasis improved after 8 weeks (from PASI 15 to PASI 5) but a vitiligo patch appeared on the penis shaft (shown in Fig. 2a, b), close to the resolved psoriasis patches. After 6 months, the penis vitiligo patch increased (shown in Fig. 3 ) in size while the subaxillary patches remained unchanged. At the last control, performed 12 months later the hypopigmented lesions had stabilized.

\section{Methodology of the Literature Search}

Literature searches in the PubMed database were conducted using the following search terms: "psoriasis" and "vitiligo" and "biologic therapy or "anti TNF-Alpha" or "anti IL-12/23" or "anti IL-17" or "anti IL-23" or "infliximab" or "adalimumab" or "etanercept" or "certolizumab pegol" or "ustekinumab" or "secukinumab," or "ixekizumab" or "brodalumab" or "guselkumab." The inclusion criterion was the English language. Articles not focused on psoriasis (i.e., biologic treatment not used to treat specifically psoriasis) were excluded.

\section{Results}

The search yielded 10 publications, described as follows, and summarized in Table 1.

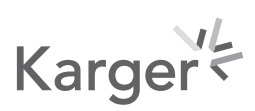




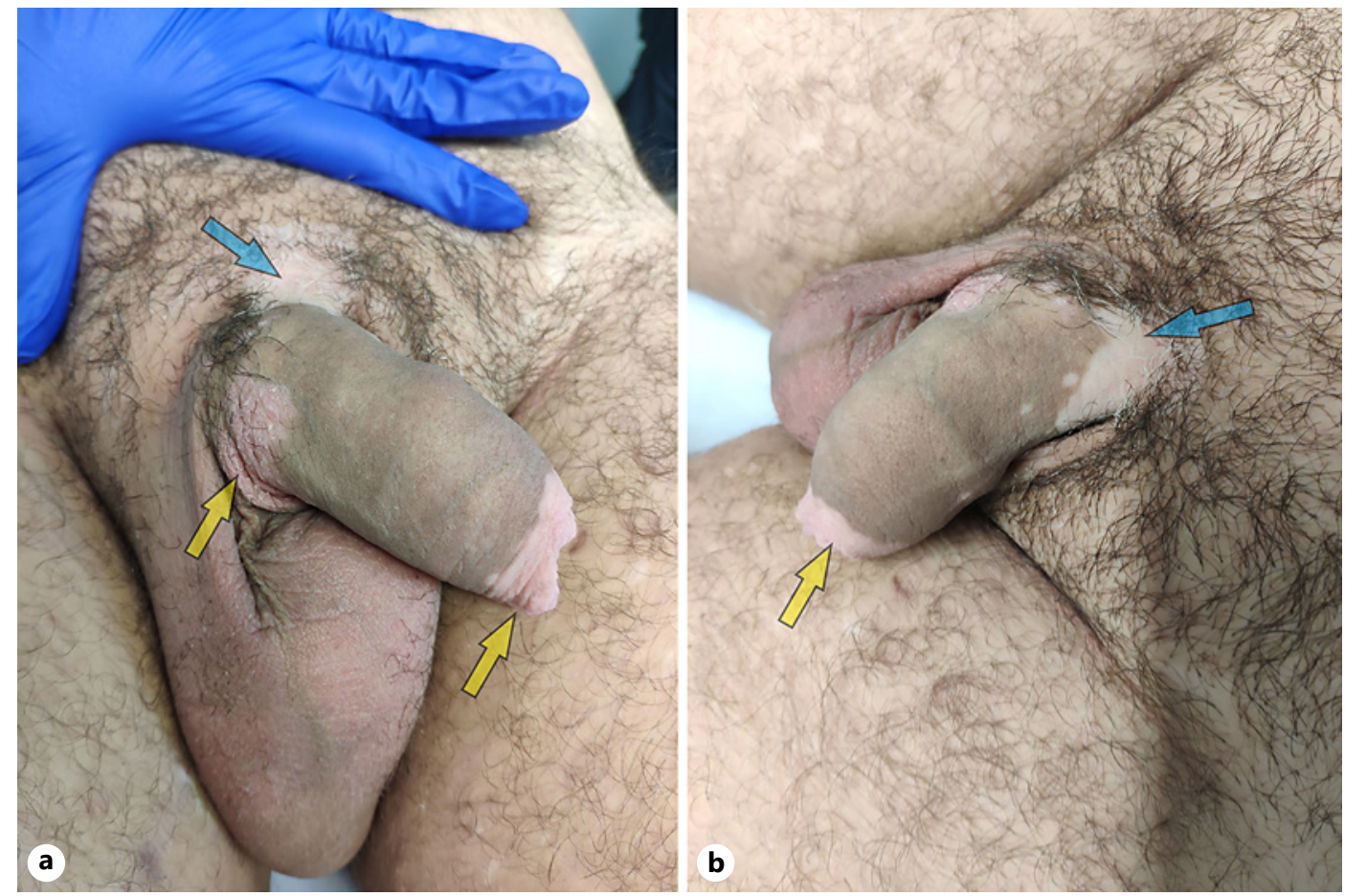

Fig. 2. a Psoriasis plaques (yellow arrows) in the resolution phase, located on the foreskin and the right-side area of the penis shaft. $\mathbf{b}$ At the base of the penis shaft (blue arrow) can be observed a large patch of vitiligo and 2 hypopigmented small maculae. These lesions appeared after 8 weeks of ustekinumab injections.

\section{Vitiligo and Anti-TNF-Alpha Class}

Six articles reported vitiligo associated with TNF-alpha inhibitors in psoriatic patients. Adalimumab is the most represented drug and reported in 4 articles. Except for one paper by Campanati et al. [2] wherein vitiligo and psoriasis improved after 24 months of treatment with etanercept, all publications described new-onset vitiligo or a progression of a pre-existence vitiligo. Lu et al. [1] described a case of nonsegmental vitiligo that appeared following infliximab infusion. The disease has been regressing for 1 year after drug interruption. Palazzo [3] described the case of a patient who suffered from vitiligo in correspondence of the psoriatic lesions after the treatment with adalimumab which resolved in 1 year switching to secukinumab. Tirado-Sánchez and Bonifaz [4] reported a case of new-onset vitiligo and bullous pemphigoid disease associated with adalimumab treatment for psoriasis. Smith and Heffernan [5] reported a case of new-onset vitiligo (nonsegmental type) that appeared in correspondence of resolute psoriatic plaques after adalimumab therapy. The lesions remained stable during biological therapy.

For certolizumab pegol, 2 cases of de novo vitiligo are reported. The disease for which the patient used the drug is not indicated [8]. According to a cohort study [10], adalimumab and infliximab seem to be the anti-TNF-alpha inhibitors with the highest risk to develop vitiligo. Overall, in the literature, the use of anti TNF-alpha drugs in repigmentation is considered ineffective [11]. Until now, no case of golimumab-induced vitiligo has been reported.

Vitiligo and Anti-IL-12/23 Class

Data from the literature are limited and reported in only 3 papers. In fact, a case of vitiligo improvement was reported in a psoriatic patient treated with ustekinumab [7]. Instead, 
Fig. 3. Increase in size of the vitiligo patch after 6 months. The lesion occupies a large part of the dorsal surface of the penis shaft.

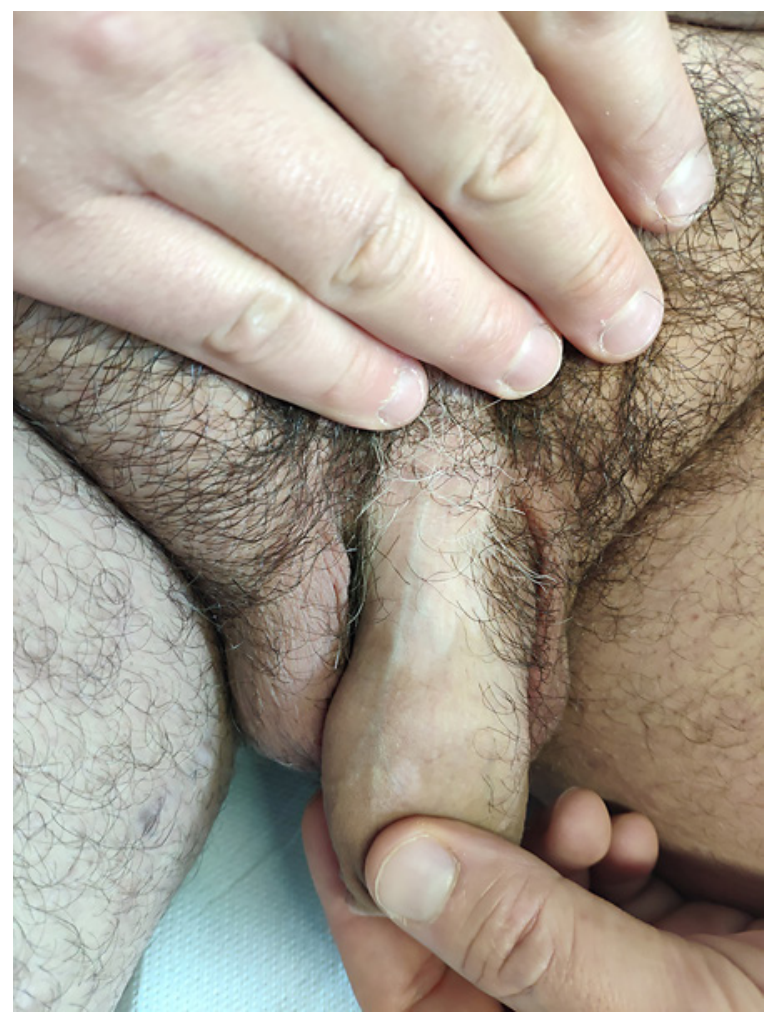

Mery-Bossard et al. [6] and Anthony et al. [8] reported, respectively, in 2 different studies, 18 total cases of ustekinumab-induced vitiligo and one case of drug-induced worsening vitiligo. In both papers, the patient's disease is not specified.

\section{Vitiligo and Anti-IL-17 Class}

Speeckaert et al. [9] reported a study on 8 patients in which secukinumab were used to treat a nonsegmental form of vitiligo. A progression of the disease was observed in 7 patients, without any sign of repigmentation. Furthermore, the authors concluded that the use of antiIL-17 was not likely to affect the pathogenetic mechanism of vitiligo, considering that IL-17 and Th17 lymphocytes do not participate in the cytotoxic destruction of melanocytes. Instead, the authors suggested that drugs acting on the differentiation imbalance of Th17 to Th17.1/ Th1 lymphocytes would be more promising. No cases are reported for brodalumab and ixekizumab.

\section{Vitiligo and Anti-IL-23 Class}

So far, there are no published reports of vitiligo induced/worse by the anti-IL-23 class.

\section{Discussion}

Psoriasis is an immune-mediated and multifactorial chronic inflammatory skin disease, with an estimated prevalence ranging from 0.51 to $11.43 \%$ in adults [12]. Data about the prevalence of vitiligo vary from 0.06 to $2.28 \%$ in the general population [12]. The prevalence of the association between these 2 diseases is not clear: it is considered a rare event, but probably, it is underestimated [12]. In fact, observational studies are very scarce, and data are 


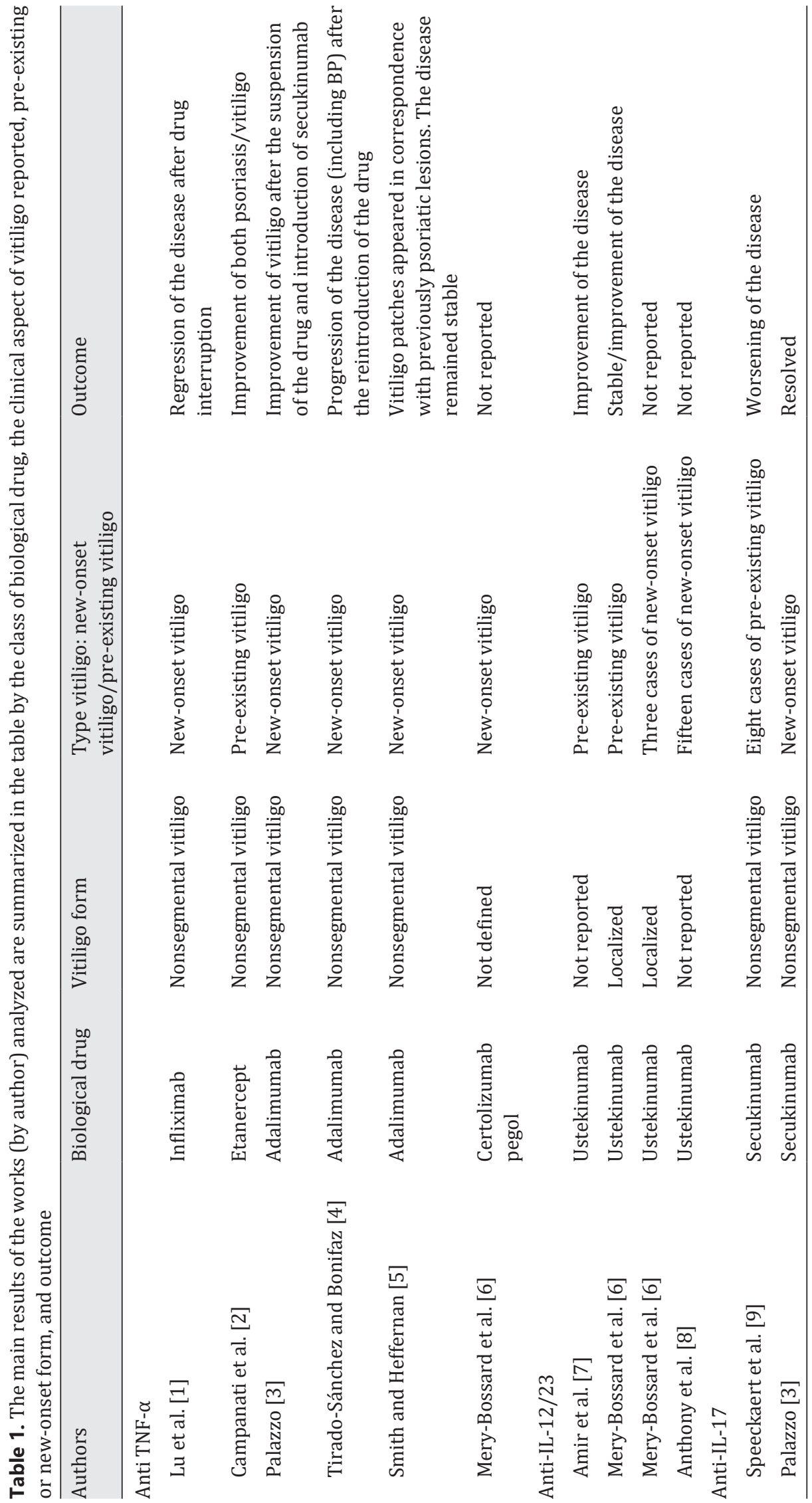


inconsistent. In clinical practice, 2 conditions usually show this association: first, psoriasis and vitiligo are present at the same time in the same patient (the so-called immune patient); second, the appearance of vitiligo is the side effect of the drugs used to treat psoriasis. In the first case, psoriasis has a strong tendency to associate with other autoimmune/immunemediated diseases. Data suggest that a patient during the lifetime has a $25 \%$ chance of developing vitiligo [13]. Similarly, psoriasis was found to be the second most associated comorbidity in patients with vitiligo [14]. These 2 disorders present a similar pathogenetic background: psoriasis and vitiligo are cell-mediated Th-1 diseases with increased TNF-alpha and interferon $\gamma$ levels [15]. Also, both share the activation of the Th17 pathway [12]. So far, it is unknown which of the 2 concurrent pathologies appears first. They can appear in the same areas or can be localized in different sites. As reported in the study [14], in almost $47 \%$ of patients with both diseases the lesions were separated, as in our case, while $34 \%$ of patient's psoriasis plaques covered the vitiligo patches [15].

Regarding vitiligo as a side effect, the literature is focused on the biological therapies and few cases are reported. Two scenarios are described: (1) vitiligo appears for the first time as a consequence of the onset of biological therapy (i.e., new-onset vitiligo) or (2) there is a preexistence vitiligo that improves or worsens as a consequence of the biological treatment. This difference has an important prognostic value: in fact, the new-onset vitiligo shows a better outcome when the biological therapy is maintained [6]. Instead, regarding a pre-existence vitiligo, the use of some biological drugs can aggravate it, inducing a progression [6], as happened to our patient.

\section{Conclusion}

The relationship among psoriasis, vitiligo, and biological drugs is still unclear. Although data are limited, this narrative review of the literature seems to show the detrimental impact of anti-TNF-alpha treatment on vitiligo. The pathogenetic mechanisms by which this class can induce or improve vitiligo are unknown. It is difficult to understand how a molecule that blocks high levels of TNF-alpha can trigger the mechanisms underlying this phenomenon. It has been proposed that the long-term use of anti TNF-alpha could create a cytokines-imbalance favoring a certain type of cytokines background that would provoke vitiligo [10].

However, it should be considered that this class of drug has been on market for the longest than other classes of biologics, and this could also explain these results. Anti-IL-12/23 drugs could be considered a trigger of vitiligo too. Except for one case reported [7], ustekinumab seems to induce vitiligo (as well as in our case) or even to worse it when it is pre-existing.

The use of anti-IL-17 seems to produce bad outcomes in pre-existing vitiligo. Conversely, the data about anti-IL-23 are scarce, possibly because this drug has been marketed only recently.

Thus, this review highlights the paucity of data on the link between psoriasis and vitiligo underlining the gaps of knowledge on this topic. Further studies aimed at highlighting the pathogenetic mechanisms underlying the 2 diseases are strongly needed.

\section{Statement of Ethics}

All the procedures adopted in the present study were in respect to the ethical standards in the World Medical Association Declaration of Helsinki. The subject gave his written informed consent to publish the present case (including publication of images).

\section{Karger'}




\section{Conflict of Interest Statement}

The authors have no conflicts of interest to declare.

\section{Funding Sources}

No funding was received.

\section{Author Contributions}

Martina Burlando concept the design of the work, draft the work, and revising it critically. Andrea Muracchioli drafts the work and contributes to the conception and the design of the work, to the acquisition and the interpretation of data. Emanuele Cozzani and Aurora Parodi have provided general support, critical review, and approval of the article.

\section{References}

1 Lu X, Gao Y, Ding Y. Vitiligo in a patient receiving infliximab for chronic plaque psoriasis. Dermatol Ther. 2019 May;32(3):e12917.

2 Campanati A, Giuliodori K, Ganzetti G, Liberati G, Offidani AM. A patient with psoriasis and vitiligo treated with etanercept. Am J Clin Dermatol. 2010;11(Suppl 1):46-8.

3 Palazzo G. Resolution of post-adalimumab vitiligo with secukinumab in a patient with psoriasis vulgaris. Oxf Med Case Rep. 2020 Jan;2020(1):omz134.

4 Tirado-Sánchez A, Bonifaz A. Simultaneous bullous pemphigoid and vitiligo associated with adalimumab therapy in a patient with psoriasis vulgaris. Indian Dermatol Online J. 2020 Mar-Apr;11(2):229-31.

5 Smith DI, Heffernan MP. Vitiligo after the resolution of psoriatic plaques during treatment with adalimumab. J Am Acad Dermatol. 2008 Feb;58(2 Suppl):S50-2.

6 Méry-Bossard L, Bagny K, Chaby G, Khemis A, Maccari F, Marotte H, et al. New-onset vitiligo and progression of pre-existing vitiligo during treatment with biological agents in chronic inflammatory diseases. J Eur Acad Dermatol Venereol. 2017 Jan;31(1):181-6.

7 Elkady A, Bonomo L, Amir Y, Vekaria AS, Guttman-Yassky E. Effective use of ustekinumab in a patient with concomitant psoriasis, vitiligo, and alopecia areata. JAAD Case Rep. 2017 Nov;3(6):477-9.

8 Anthony N, Bourneau-Martin D, Ghamrawi S, Lagarce L, Babin M, Briet M. Drug-induced vitiligo: a case/ non-case study in Vigibase ${ }^{\circ}$, the WHO pharmacovigilance database. Fundam Clin Pharmacol. 2020 Dec;34(6): 736-42.

9 Speeckaert R, Mylle S, van Geel N. IL-17A is not a treatment target in progressive vitiligo. Pigment Cell Melanoma Res. 2019 Nov;32(6):842-7.

10 Bae JM, Kim M, Lee HH, Kim KJ, Shin H, Ju HJ, et al. Increased risk of vitiligo following anti-tumor necrosis factor therapy: a 10-year population-based cohort study. J Invest Dermatol. 2018 Apr;138(4):768-74.

11 Alghamdi KM, Khurrum H, Taieb A, Ezzedine K. Treatment of generalized vitiligo with anti-TNF- $\alpha$ agents. J Drugs Dermatol. 2012 Apr;11(4):534-9.

12 Yen H, Chi CC. Association between psoriasis and vitiligo: a systematic review and meta-analysis. Am J Clin Dermatol. 2019 Feb;20(1):31-40.

13 Masood S, Sajid S, Jafferani A, Tabassum S, Ansar S. Multiple autoimmune syndromes associated with psoriasis: a rare clinical presentation. Oman Med J. 2014 Mar;29(2):130-1.

14 Sheth VM, Guo Y, Qureshi AA. Comorbidities associated with vitiligo: a ten-year retrospective study. Dermatology. 2013;227(4):311-5.

15 Sandhu K, Kaur I, Kumar B. Psoriasis and vitiligo. J Am Acad Dermatol. 2004 Jul;51(1):149-50. 